\title{
Efficacy of online and face-to-face cognitive behavioral therapy in the treatment of neurological insomnia: a systematic review and meta-analysis
}

\author{
Hang Yu\#, Yuanchao Zhang", Qingchang Liu, Ruoxin Yan \\ Neurology Medicine Center, The Seventh Affiliated Hospital of Sun Yat-sen University, Shenzhen, China \\ Contributions: (I) Conception and design: H Yu, Y Zhang; (II) Administrative support: Q Liu; (III) Provision of study materials or patients: H Yu, \\ Y Zhang, R Yan; (IV) Collection and assembly of data: All authors; (V) Data analysis and interpretation: Y Zhang, Q Liu, R Yan; (VI) Manuscript \\ writing: All authors; (VII) Final approval of manuscript: All authors. \\ "These authors contributed equally to this work. \\ Correspondence to: Hang Yu. Neurology Medicine Center, The Seventh Affiliated Hospital of Sun Yat-sen University, 628 Zhenyuan Road, Shenzhen, \\ China. Email: 402203504@163.com.
}

\begin{abstract}
Background The treatment of insomnia mainly includes drug therapy and non-drug therapy (cognitive behavioral therapy [CBT]). Traditional face-to-face CBT is affected by factors such as location, time, and treatment cost, making the treatment impossible to implement effectively. With the continuous development of network technology, internet-based CBT (ICBT) has been widely used due to the advantages of time and location.

Methods: It searched the China National Knowledge Internet (CNKI) database (1979- Apr 2021), China Biomedical Literature Database (1994 - Apr 2021), Cochrane Library (2005 - Apr 2021), Medline (1948Apr 2021), and Embase (Jan 1966 - Apr 2021). Chinese and English databases were searched using the following terms: internet-based cognitive behavioral therapy, cognitive behavioral therapy, sleep problems, and sleep disorders. Meta-analysis was performed using RevMan 5.3 and Stata SE 12.0 software provided by the Cochrane collaboration.

Results: A total of 14 randomized controlled trials were included in this study. Of these, 10 described the correct random allocation method, 6 described the allocation scheme in detail, and 1 article used the blind method. Sleep onset latency after ICBT was much shorter than that of the control group [mean difference (MD): $-12.27,95 \%$ confidence interval (CI): -16.43 to $-9.90, \mathrm{P}<0.01]$. Total sleep time after ICBT was much longer than that of the control group (MD: 38.67, 95\% CI: 34.70-42.65, P<0.01). Sleep efficacy after ICBT was significantly higher in contrast with the control group (MD: 13.28, 95\% CI: 10.49-16.06, P<0.01). Anxiety and depression levels after the ICBT were significantly lower than those in the control group $(\mathrm{P}<0.01)$. Discussion: Meta-analysis was adopted to confirm that ICBT can greatly improve the sleep parameters of patients with insomnia, and it was found to have a relieving effect on patients with anxiety and depression.
\end{abstract}

Keywords: Online cognitive behavioral therapy; face-to-face cognitive behavioral therapy; insomnia; metaanalysis

Submitted Aug 08, 2021. Accepted for publication Sep 24, 2021.

doi: $10.21037 / \mathrm{apm}-21-2387$

View this article at: https://dx.doi.org/10.21037/apm-21-2387

\section{Introduction}

Insomnia usually refers to a subjective experience in which the patient is not satisfied with the sleep time or quality and affects daytime social functions $(1,2)$. Adequate sleep makes people relax physically and mentally. However, nearly $15 \%$ of adults worldwide suffer from insomnia. The 
2015 China Sleep Index report shows that about 31.2\% of people in China have serious sleep problems (3). The causes of insomnia include six aspects: environmental reasons, common sudden changes in sleeping environment; individual factors, bad living habits, such as drinking tea, coffee, and smoking before going to bed; physical reasons, any physical discomfort can lead to insomnia; mental factors, including opportunistic insomnia caused by excitement and anxiety caused by a special event; emotional factors, emotion out of control can cause changes in the state of mind, which will especially show up when the mood is unstable; sleeping pills or abstinence reactions of alcoholics $(4,5)$. About $41 \%$ of insomnia patients have symptoms of depression (6). In the second edition of the International Classification of Sleep Disorders, insomnia is divided into primary insomnia and secondary insomnia (7). Patients with insomnia also suffer from lack of energy, obesity, hair loss, and memory loss.

Chronic insomnia can affect the quality of life, occupational functioning and health of patients, and can cause psychological problems. However, most patients with insomnia do not receive safe and effective treatment (8). The current clinical treatment of insomnia is divided into drug therapy and non-drug therapy. Drug therapy mainly treats insomnia by inhibiting brain activity to induce and promote current sleep (9-11). Drugs have no effect on maintenance factors, so it is difficult to cure chronic insomnia. Moreover, patients with long-term use of drug treatment tolerance and dependence problems can't fundamentally solve the pain of insomnia patients. Some people with insomnia refuse to take sleeping pills for treatment because of psychological rejection or physical reaction to the side effects of drugs.

Cognitive behavioral therapy (CBT) is a non-drug therapy that helps people cope with insomnia by eliminating risk factors for insomnia through behavioral and cognitive interventions $(12,13)$. It has the advantages of a high cure rate and wide application range (14), and it is recommended as the first-line treatment for insomnia in the guidelines of the American Medical Association (15). However, traditional face-to-face CBT has disadvantages, such as high labor costs and low economic benefits. With the advancement of science and technology, internet-based online CBT (ICBT) through artificial intelligence terminals has lowered the threshold of CBT and helped optimize diagnosis and treatment. In recent years, ICBT has been gradually promoted in China, but there are few clinical studies on ICBT for the treatment of insomnia.

At present, the research conclusions about the efficacy of online cognitive behavioral therapy on insomnia can't be unified, so it is necessary to include more high-quality randomized controlled trials for systematic evaluation and meta-analysis. Moreover, the difference in curative effect between online cognitive behavioral therapy and traditional face-to-face cognitive behavioral therapy is still unclear, which needs further exploration. The innovation of this study is to compare the efficacy of online cognitive behavioral therapy and traditional face-to-face cognitive behavioral therapy by systematic evaluation and meta-analysis, so as to provide scientific evidence-based basis for the clinical promotion of online cognitive behavioral therapy.

We present the following article in accordance with the PRISMA reporting checklist (available at https://dx.doi. org/10.21037/apm-21-2387).

\section{Methods}

\section{Article retrieval}

We searched the China National Knowledge Internet (CNKI) database (1979- Apr 2021), China Biomedical Literature Database (1994 - Apr 2021), Cochrane Library (2005 - Apr 2021), Medline (1948-Apr 2021), and Embase (Jan 1966 - Apr 2021). The search articles were published RCTs of ICBT for the treatment of neurological insomnia. The obtained articles were manually searched in the journals. The compound logic retrieval Boolean logic retrieval method was adopted to select related articles. The Chinese databases were searched using a combination of the following terms: internet-based cognitive behavioral therapy, cognitive behavioral therapy, insomnia, sleep disorders, and sleep problems. The English databases were searched using the following terms: internet-based cognitive behavioral therapy, cognitive behavioral therapy, sleep problems, and sleep disorders as search terms. The meta-analysis was performed using RevMan 5.3 and Stata SE 12.0 software provided by the Cochrane collaboration.

The search terms were randomly combined, and the initially retrieved articles were screened for the first time through reading titles and abstracts. Inconsistent articles were excluded. A second screening was conducted according to the inclusion and exclusion criteria, and the search engine was used to trace the included articles. Finally, the full texts of the included articles were read for a third screening to evaluate the quality of the articles.

\section{Inclusion and exclusion criteria of the articles}

The inclusion criteria were as follows: articles designed 
as RCTs, articles with participants meeting the diagnostic criteria for insomnia in the International Classification of Sleep Disorders (16), participants with insomnia symptoms $>3$ times a week for more than 4 weeks, articles with intervention measures in the experimental group of ICBT, and articles with participants who had complete basic clinical data and observational indicators.

The exclusion criteria were as follows: articles with ICBT intervention treatment in the experimental group $<4$ weeks, articles mentioning patients with $>2$ meetings with the treating physician when receiving ICBT, articles in which patients had combined mental illness, the same article which were published in multiple journals, and conference proceedings, review articles, research reports, lectures, and other documents.

\section{Observational indicators}

The primary research indicators were sleep onset latency (SOL), total sleep time (TST), sleep efficiency (SE), and wakefulness after sleep onset (WASO).

The secondary research indicators were time in bed (TIB), number of awakenings after falling asleep (NOA), insomnia severity index (ISI), anxiety, and depression.

\section{Data extraction}

The data was extracted independently by 2 experts using Microsoft Excel. When the experts showed inconsistent views, a consistent conclusion was obtained through discussion. The data extracted for the obtained articles included basic information of the articles (first author, title, time, sample size, male-to-female ratio, average age, and diagnostic criteria for insomnia), intervention measures for the experimental group (including sleep restriction, cognitive therapy, relaxation therapy, and sleep health education) and control group, the number of cases lost to follow up in the experimental group and control group, and the extracted observational indicators.

\section{Assessment of quality and risk of bias}

Two researchers assessed the risk of bias. Any inconsistencies between the researchers were resolved by discussion or arbitration with a third expert. The Cochrane Collaboration's tool for assessing risk of bias was used to evaluate the bias risk of the included RCTs, including whether the random allocation method was correct, whether the allocation plan was hidden, and whether the method was correct; whether or not to use the blinding method for research objects, treatment plan, and research results; whether the research results were selectively reported; and whether the research data were complete. The above items were considered to be high-risk bias, low-risk bias, and unclear risk bias.

\section{Statistical analysis}

Statistical analysis was performed using Stata SE 12.0 software (College Station, Texas, USA). RevMan 5.3 software was used to draw the risk of bias assessment chart to assess the risk bias of the included articles. The results of continuous variables (SOL, TST, SE, WASO, TIB, and ISI) were expressed as the mean difference (MD), anxiety and depression scores were expressed as the standardized MD (SMD), and non-continuous variables were expressed as odds ratio (OR). Each effect was represented by a $95 \%$ confidence interval (CI). $\mathrm{I}^{2}$ is a warranty that measures the degree of variation between effects of multiple studies, describing the percentage of variation due to inter-study variation. When $\mathrm{P}>0.01$ and $\mathrm{I}^{2}<50 \%$, a fixed-effects model (FEM) was used for the meta-analysis. When $\mathrm{P}<0.01$ and $\mathrm{I}^{2}>50 \%$, a randomeffects model (REM) was used for the meta-analysis.

\section{Results}

\section{Article retrieval and basic information of included articles}

862 documents were obtained by database search, 395 were obtained by manual search of periodicals, 126 repeatedly published documents were eliminated, 85 unqualified documents were excluded, and 84 were excluded for other reasons, leaving 962 documents. After reading the title and abstract of the article, 854 articles were excluded. Seventythree review articles and research reports were excluded, leaving 35 articles. After reading the full text carefully, 15 articles of non-randomized controlled trials and 6 articles with insufficient observation indexes were excluded. Finally, 14 articles were included in the study (Figure 1).

Fourteen articles met the inclusion criteria, and there were 2,263 patient cases. Among the 14 articles, they were all small-sample studies, with the sample size ranging from 67 to 270 . The age of the participants was $>20$ years old. The 14 articles detailed the sample size, sex ratio, age, intervention measures, and follow-up time. Table 1 shows the basic characteristics of the included articles. 


\section{Identification of studies via databases and registers}

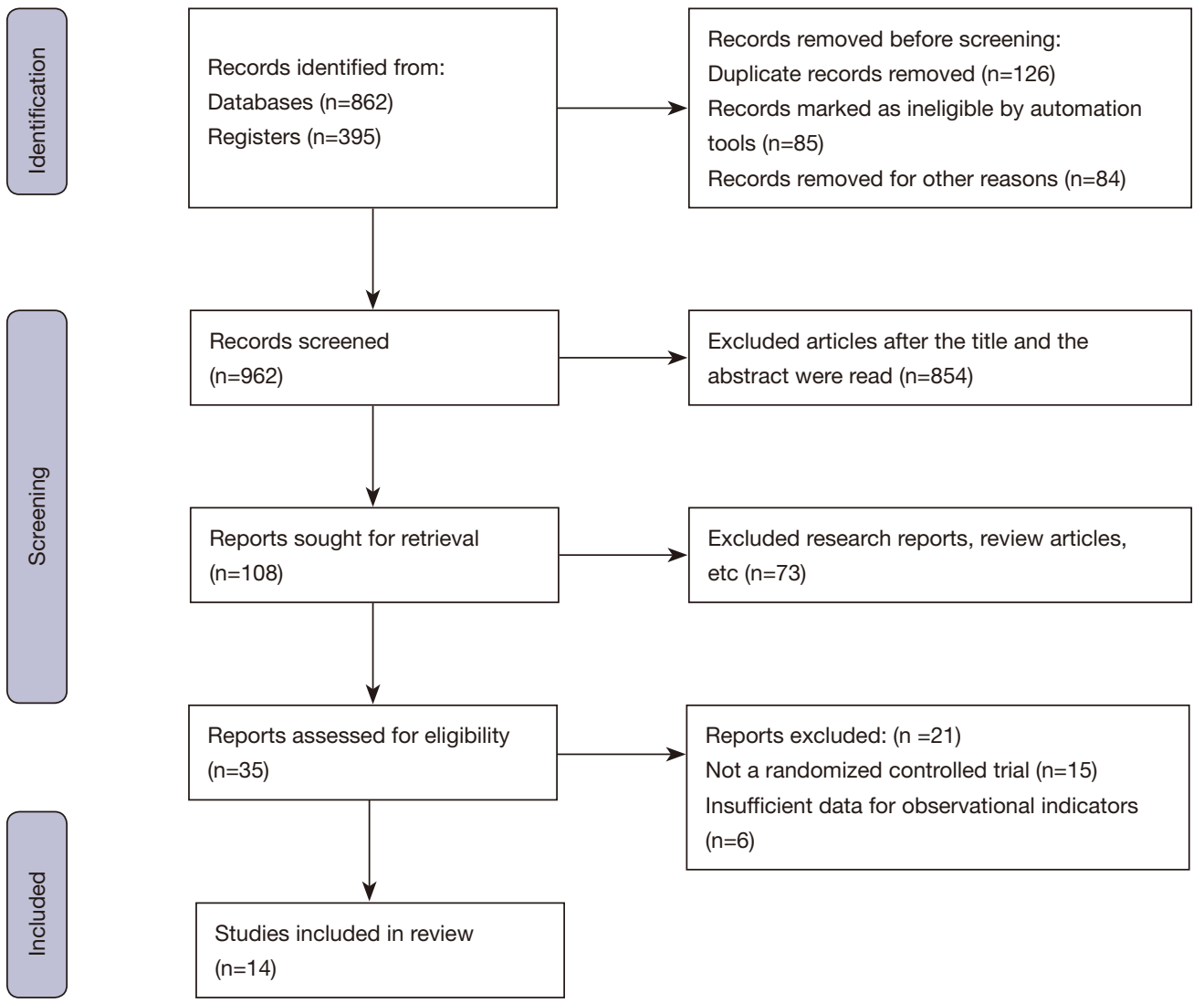

Figure 1 Flowchart of the article retrieval process.

\section{Risk bias assessment of the included articles}

Figures 2,3 show the results of multiple risk bias evaluations of the included articles drawn by RevMan 5.3 software. Among the 14 RCTs in this study, 10 described the correct random allocation method (accounting for $71.43 \%$ ), 6 described the hidden allocation plan in detail (accounting for $42.86 \%$ ), 1 article used the blinding method (accounting for $7.14 \%$ ), and others used no blinding method.

\section{Meta-analysis results of $S O L$}

The forest map of SOL is shown in Figure 4. The MD and SMD of SOL were described in detail in 12 articles. Relevant data of 12 articles were extracted, and the heterogeneity analysis was performed on the SOL of patients with insomnia. The results showed that $\chi^{2}$-test: 120.58 , degrees of freedom (df): $11, \mathrm{I}^{2}: 91 \%>50 \%$, and $\mathrm{P}<0.01$, indicating that there was obvious heterogeneity. Statistical analysis using a REM found that the SOL after ICBT was significantly shorter than that of the control group (MD: $-12.27,95 \%$ CI: -16.43 to $-9.90, \mathrm{P}<0.01)$.

\section{Meta-analysis results of TST}

The forest map of TST is shown in Figure 5. Based on the MD and SMD of TST in 14 articles, relevant data were extracted from 8 articles to analyze the heterogeneity of TST in patients with insomnia. The results showed that $\chi^{2}-$ test: 19.17 , df: $13, \mathrm{I}^{2}: 32 \%<50 \%$, and $\mathrm{P}>0.01$, indicating that there was no heterogeneity. Statistical analysis using a FEM found that the TST after ICBT was significantly longer than the control group (MD: 38.67, 95\% CI: 34.70-42.65, $\mathrm{P}<0.01)$. 


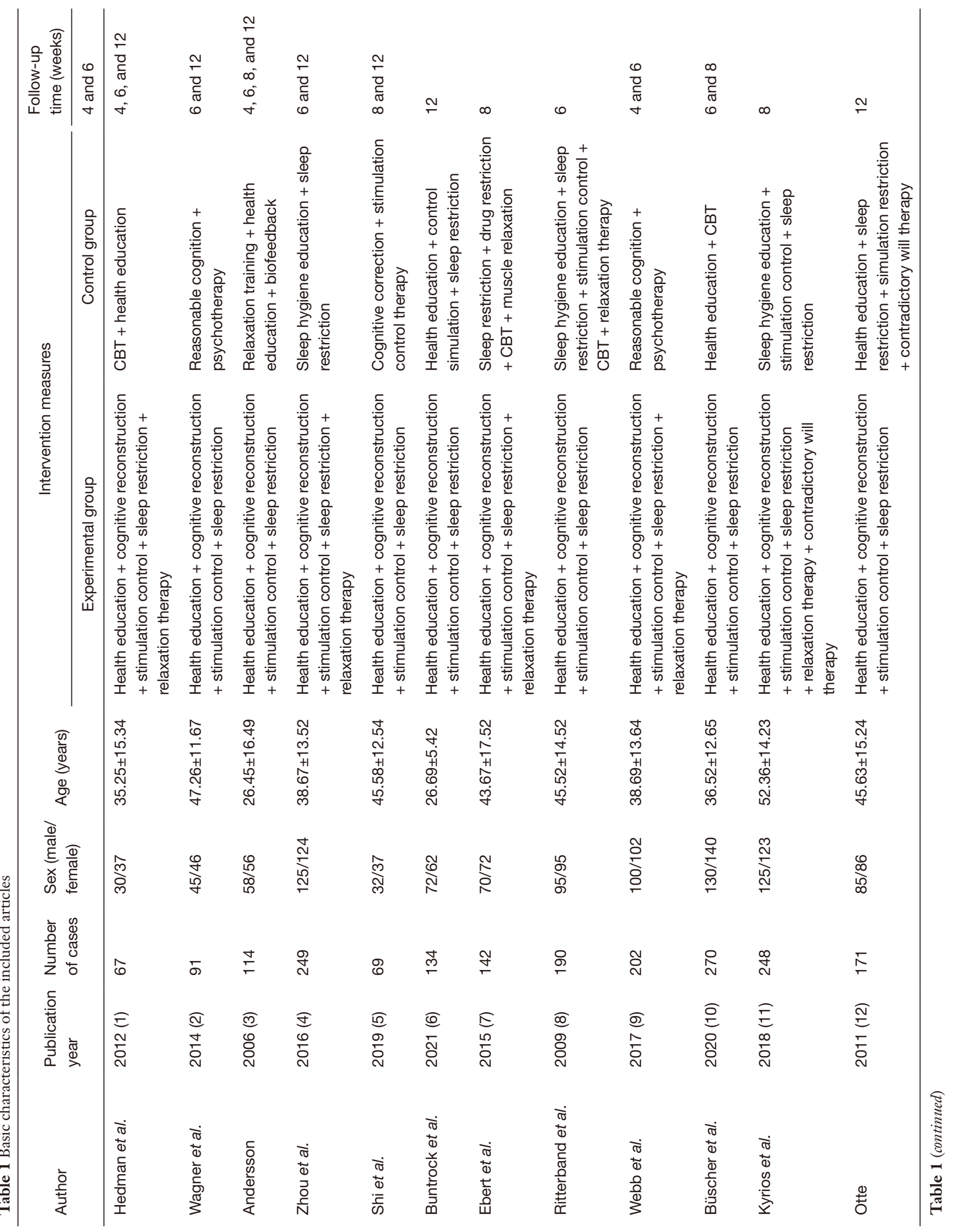




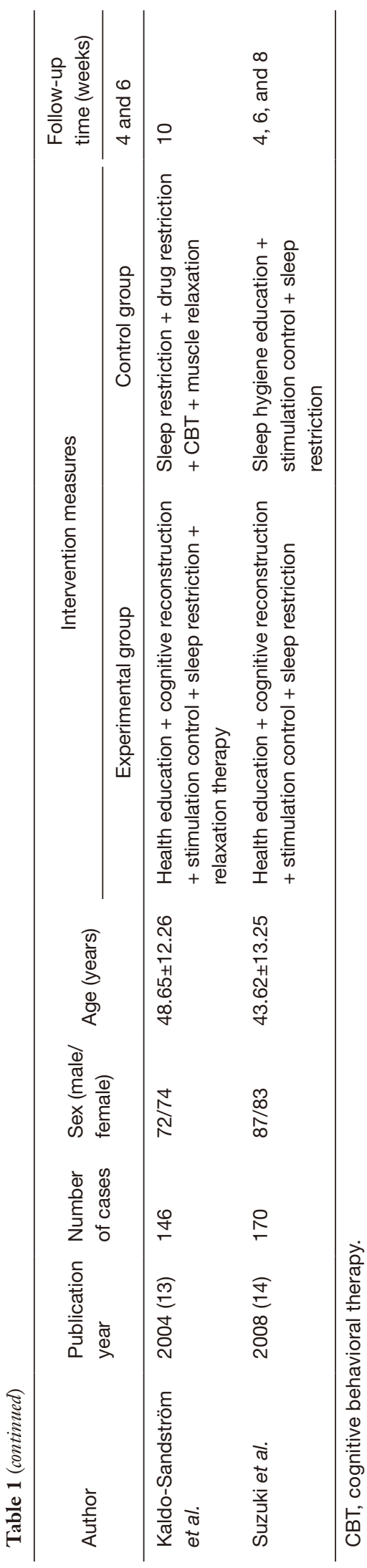

\section{Meta-analysis results of SE}

The forest map of SE is shown in Figure 6. Based on the MD and SMD of SE in 12 articles, relevant data were extracted from 8 articles to analyze the heterogeneity of SE in patients with insomnia. The results showed that $\chi^{2}$-test: 81.64 , df: 11 , $\mathrm{I}^{2}: 87 \%>50 \%$, and $\mathrm{P}<0.01$, indicating that there was obvious heterogeneity. Statistical analysis using a REM found that the SE after ICBT was significantly higher than the control group (MD: 13.28, 95\% CI: 10.49-16.06, $\mathrm{P}<0.01$ ).

\section{Meta-analysis results of wake-up time after sleep}

The forest map of wake-up time after sleep is shown in Figure 7. Based on the MD and SMD of SE in 10 articles, relevant data were extracted from 10 articles to analyze the heterogeneity of wake-up time after sleep in patients with insomnia. The results showed that $\chi^{2}$-test: 25.38 , df: $9, \mathrm{I}^{2}$ : $65 \%>50 \%$, and $\mathrm{P}<0.01$, indicating that there was obvious heterogeneity. Statistical analysis using a REM found that the wake-up time after sleep after ICBT was significantly shorter than the control group (MD: 16.91, 95\% CI: -19.89 to $-13.94, \mathrm{P}<0.01)$.

\section{Meta-analysis results of $T I B$}

The forest map of TIB is shown in Figure 8. Based on the MD and SMD of TIB in 6 articles, relevant data were extracted from 6 articles to analyze the heterogeneity of TIB in patients with insomnia. The results showed that $\chi^{2}-$ test: 8.64 , df: $5, \mathrm{I}^{2}: 42 \%<50 \%$, and $\mathrm{P}>0.01$, indicating that there was no obvious heterogeneity. Statistical analysis using a FEM found that the TIB after ICBT was significantly shorter than the control group (MD: $-16.94,95 \% \mathrm{CI}$ : -24.16 to -9.71 , and $\mathrm{P}<0.01$ ).

\section{Meta-analysis results of $\mathrm{NOA}$}

The forest map of NOA is shown in Figure 9. Based on the MD and SMD of NOA in 8 articles, relevant data were extracted from 8 articles to analyze the heterogeneity of NOA in patients with insomnia. The results showed that $\chi^{2}$ test: 1.68 , df: $7, \mathrm{I}^{2}: 0 \%<50 \%$, and $\mathrm{P}>0.01$, indicating that there was no obvious heterogeneity. Statistical analysis using a FEM found that the NOA after ICBT was significantly shorter than the control group (OR: 0.33, 95\% CI: $0.17-$ $0.67, \mathrm{P}<0.01)$. 


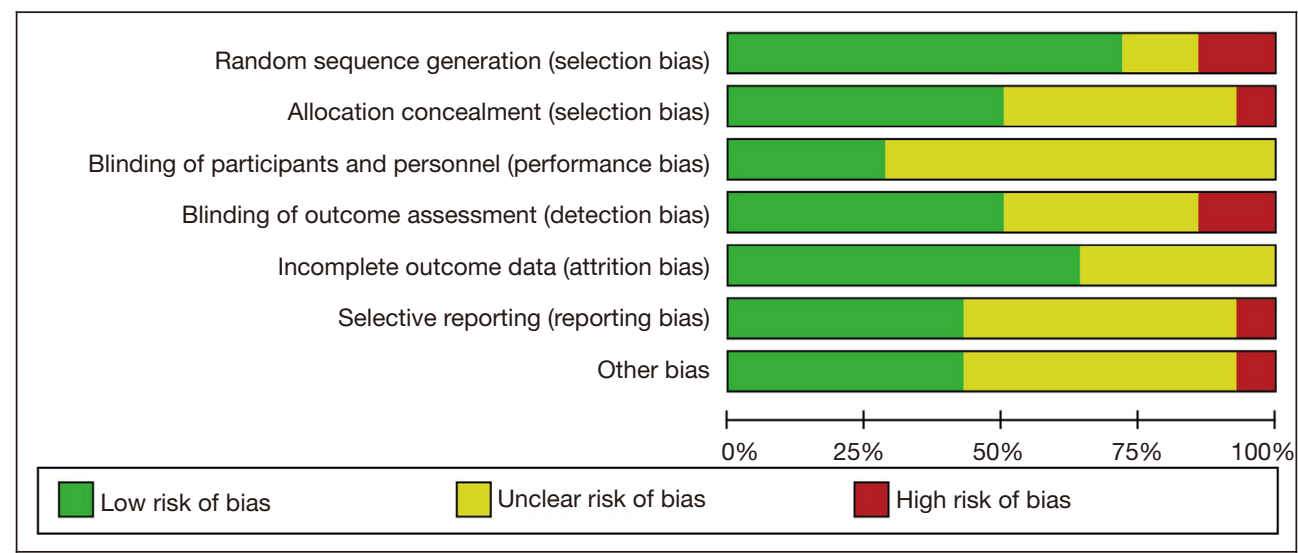

Figure 2 Assessment of risk bias of the included articles.

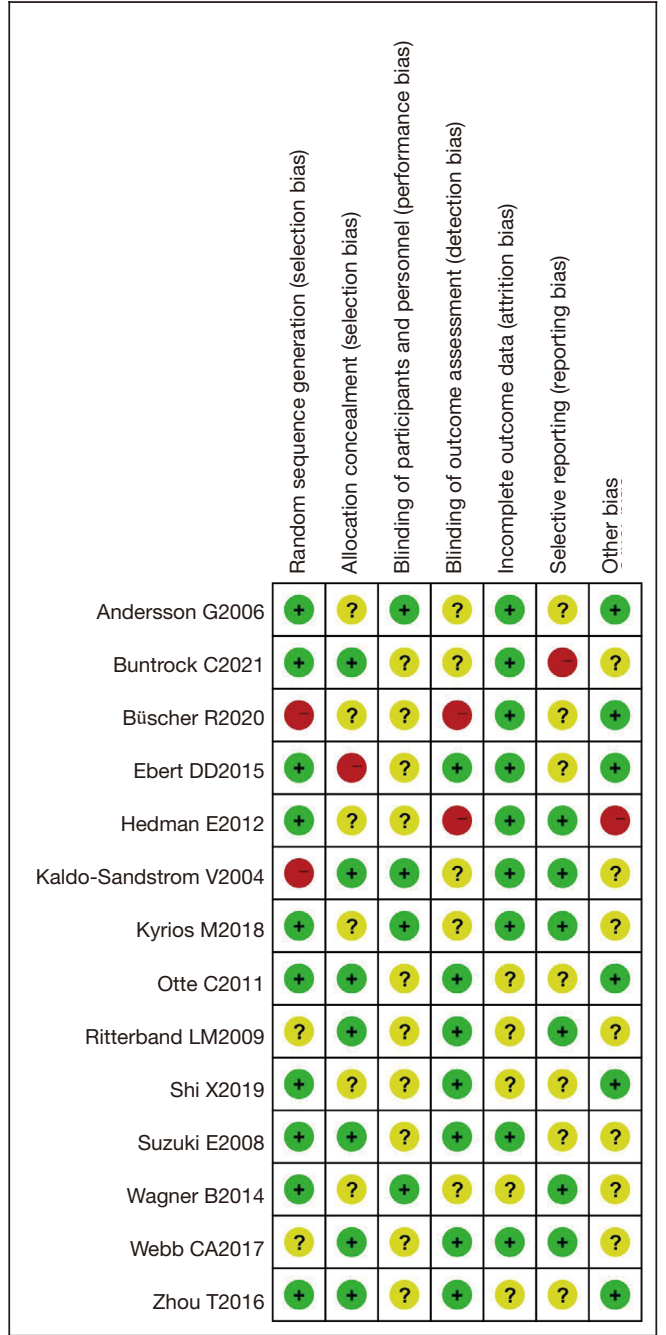

Figure 3 Multiple studies in the articles correspond to the multiple risk bias evaluation results. +, -, and ? refer to low-risk bias, highrisk bias, and unclear risk bias, respectively.

\section{Meta-analysis results of ISI}

The forest map of ISI is shown in Figure 10. Based on the MD and SMD of ISI in 8 articles, relevant data were extracted from 8 articles to analyze the heterogeneity of ISI in patients with insomnia. The results showed that $\chi^{2}$-test: 79.84, df: $7, \mathrm{I}^{2}: 91 \%>50 \%$, and $\mathrm{P}<0.01$, indicating that there was obvious heterogeneity. Statistical analysis using a REM found that the ISI after ICBT was significantly smaller than the control group (MD: $-6.34,95 \%$ CI: -8.24 to -4.44 , $\mathrm{P}<0.01)$.

\section{Meta-analysis results of anxiety level}

The forest map of anxiety level is shown in Figure 11. Based on the MD and SMD of anxiety level in 6 articles, relevant data were extracted from 6 articles to analyze the heterogeneity of anxiety level in patients with insomnia. The results showed that $\chi^{2}$-test: 26.56 , df: $5, \mathrm{I}^{2}: 81 \%>50 \%$, and $\mathrm{P}<0.01$, indicating that there was obvious heterogeneity. Statistical analysis using a REM found that the anxiety level after ICBT was significantly lower than the control group (SMD: $-0.56,95 \%$ CI: -0.84 to $-0.29, \mathrm{P}<0.01$ ).

\section{Meta-analysis results of depression level}

The forest map of depression level is shown in Figure 12. Based on the MD and SMD of depression level in 8 articles, relevant data were extracted from 8 articles to analyze the heterogeneity of depression level in patients with insomnia. The results showed that $\chi^{2}$-test: 12.28 , df: $7, \mathrm{I}^{2}$ : $43 \%<50 \%$, and $\mathrm{P}>0.01$, indicating that there was no obvious heterogeneity. Statistical analysis using a FEM found that 


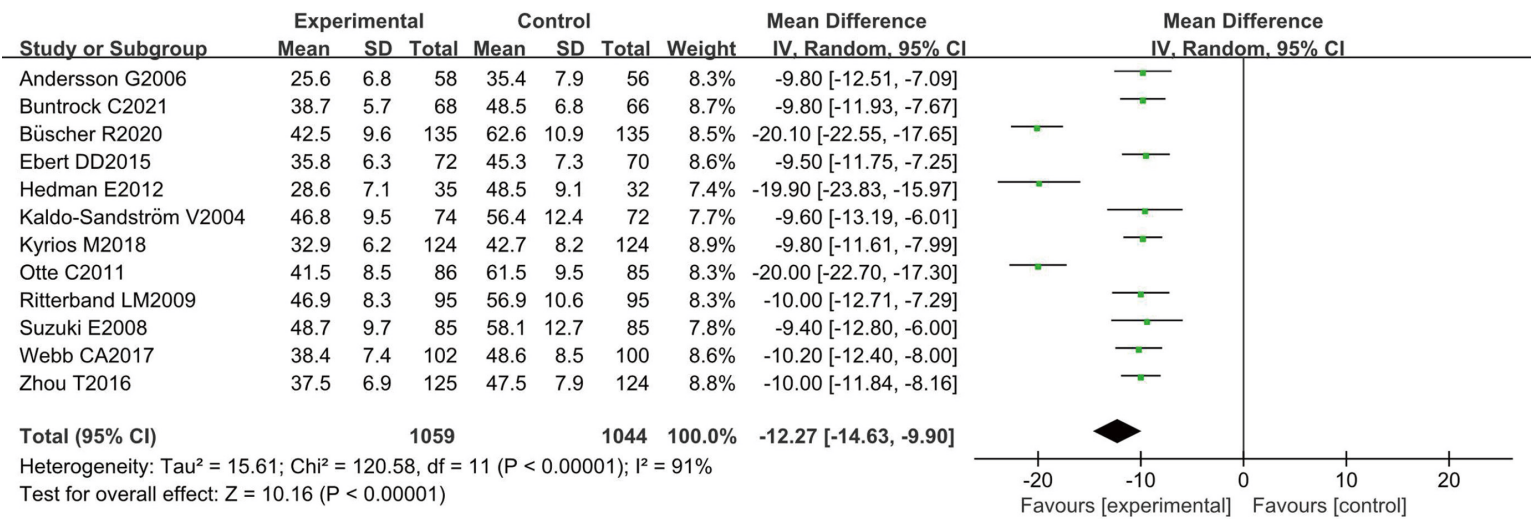

Figure 4 Sleep onset latency forest map.

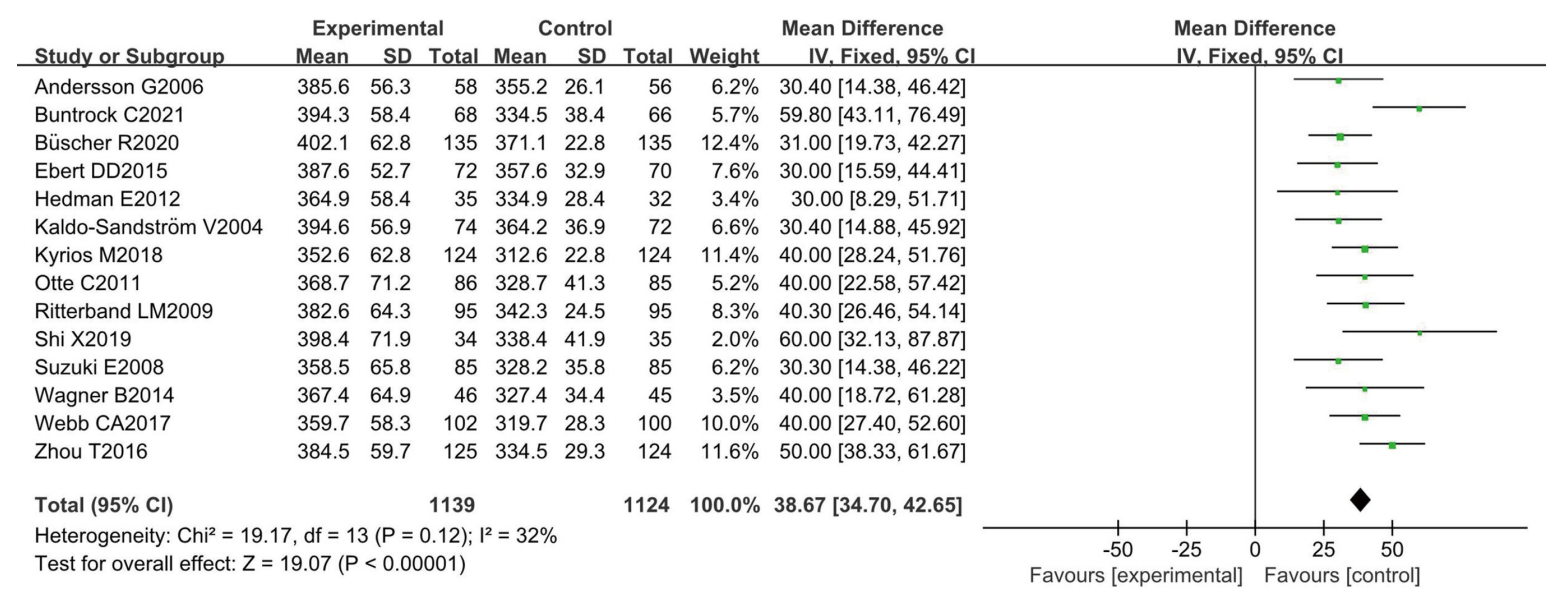

Figure 5 Total sleep time forest map.

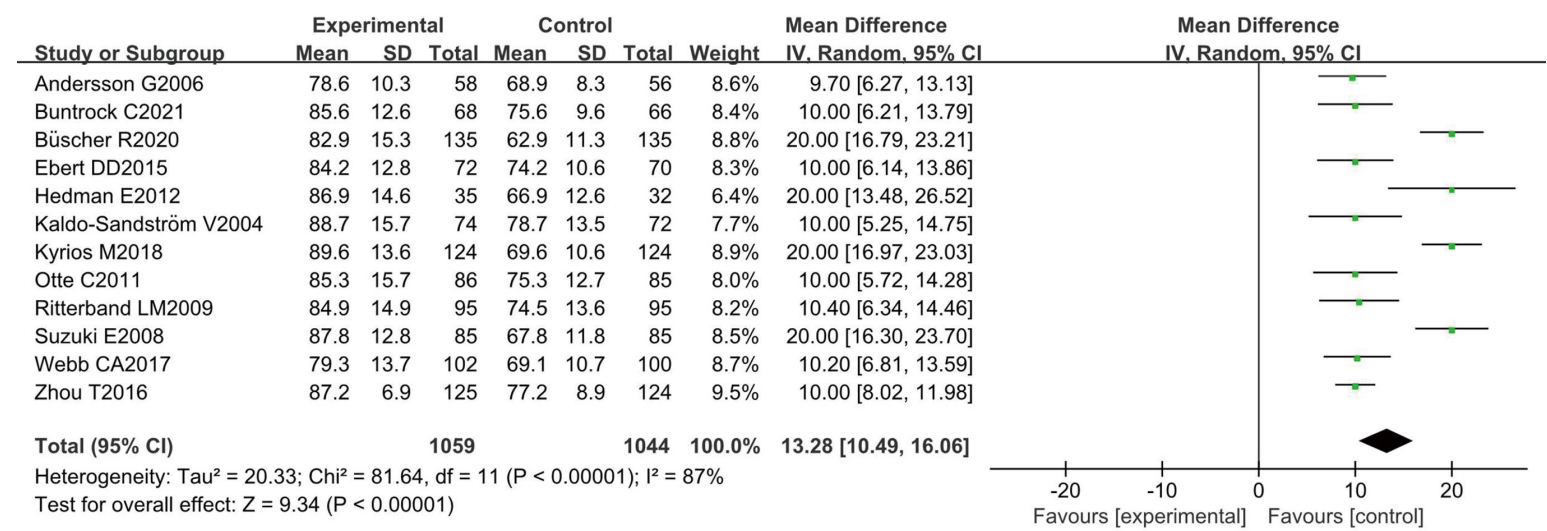

Figure 6 Sleep efficiency forest map. 


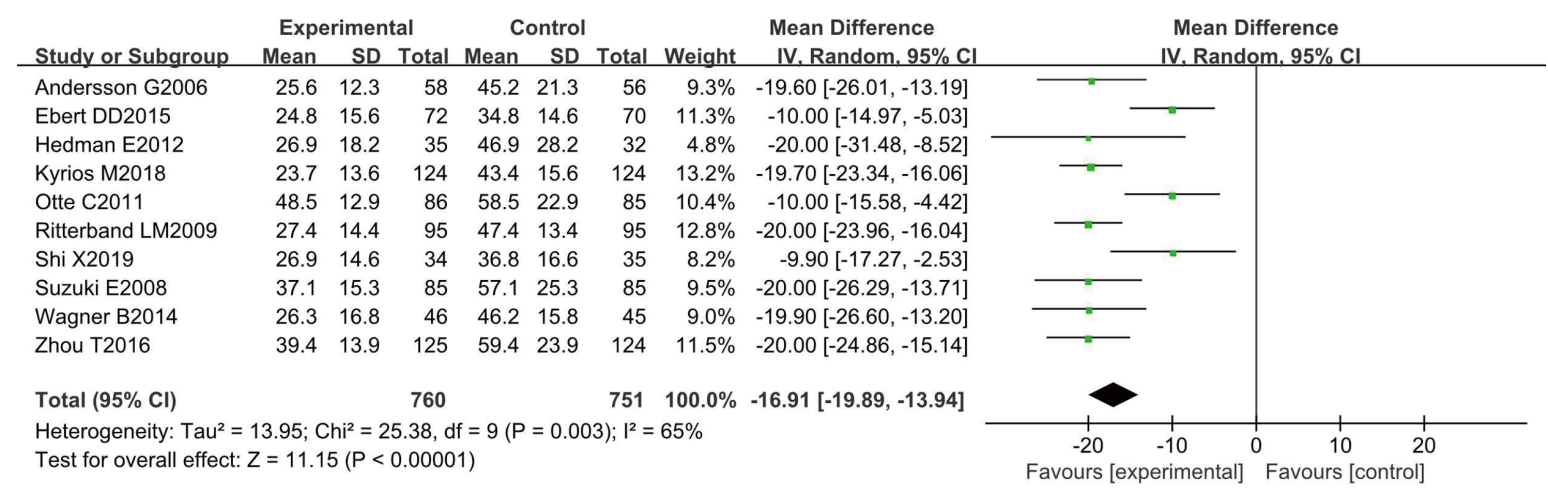

Figure 7 Wake-up after sleep forest map.

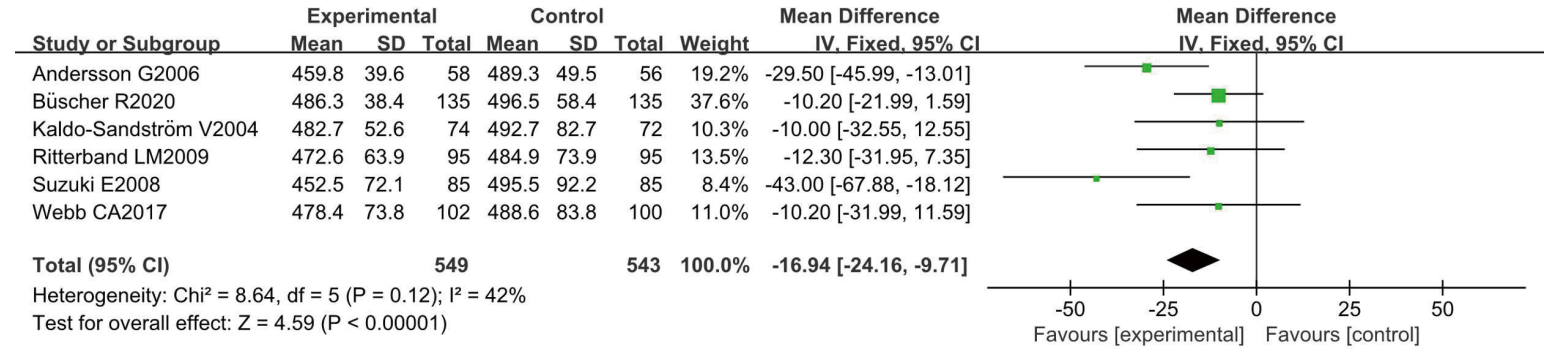

Figure 8 Time in bed forest map.

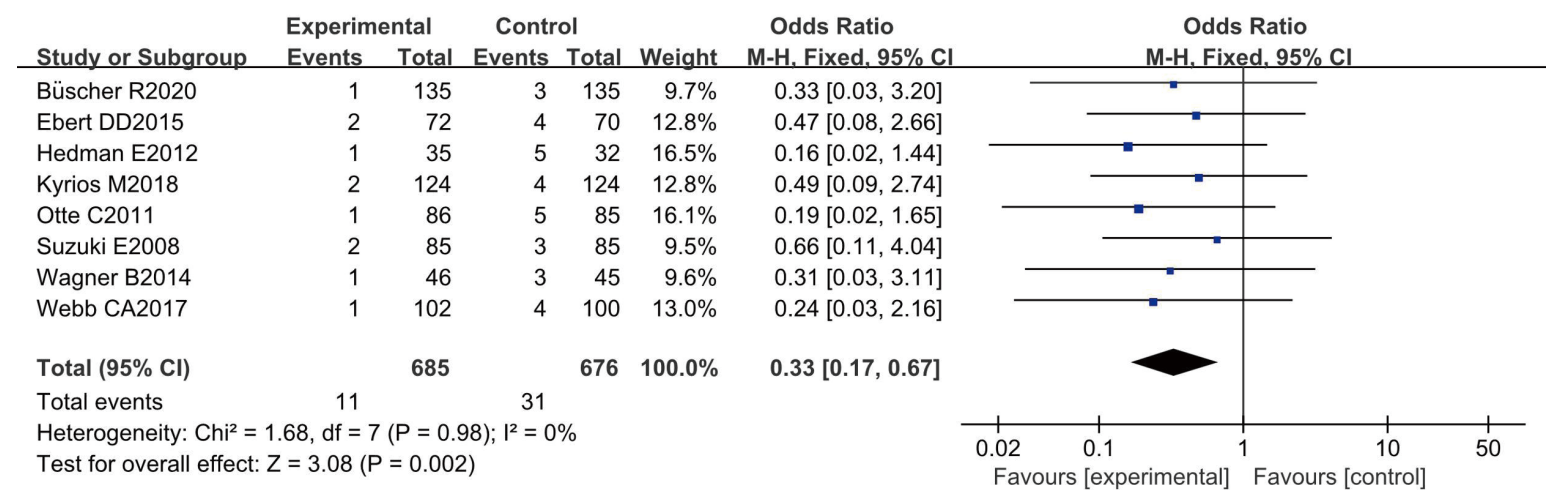

Figure 9 Number of awakenings after falling asleep forest map.

the depression level after ICBT was significantly lower than the control group (SMD: $-0.83,95 \% \mathrm{CI}:-0.94$ to -0.71 , $\mathrm{P}<0.01)$.

\section{Analysis of publication bias}

A funnel chart of publication bias in the included articles for TST is shown in Figure 13. Circles in the chart that are concentrated on the midline and roughly symmetrical to the midline indicate that there was no bias in the publication of the included articles, and that the conclusions drawn were relatively reliable.

\section{Discussion}

A total of 14 articles were included in the systematic review 


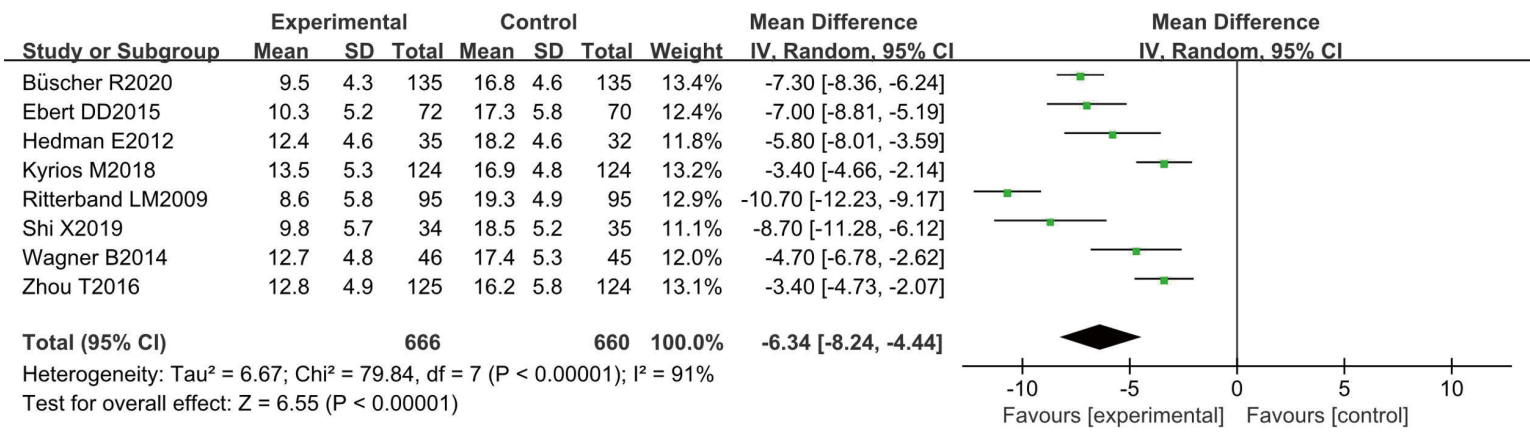

Figure 10 Insomnia severity index forest map.

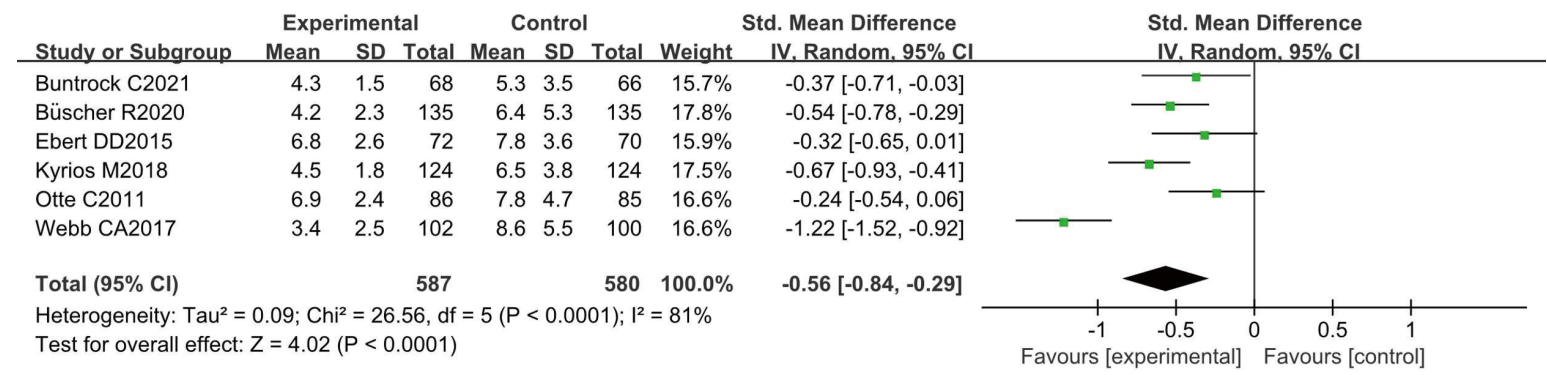

Figure 11 Anxiety level forest map.

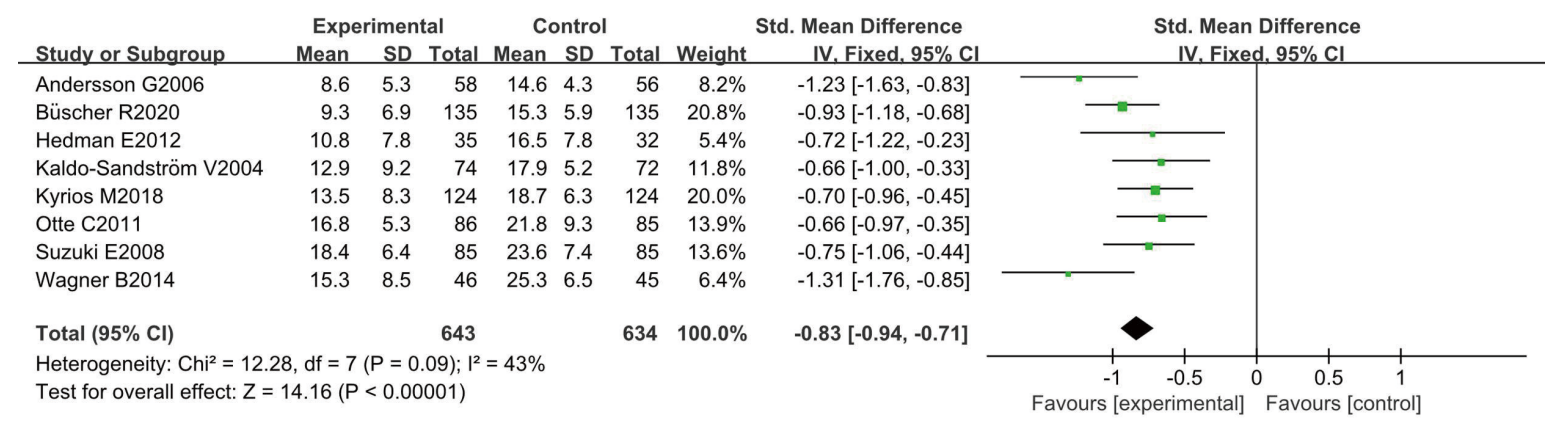

Figure 12 Depression level forest map.

of this study, with the purpose of evaluating the efficacy of ICBT and traditional face-to-face CBT on patients with neurological insomnia. There were 10 articles describing the correct random allocation method, 6 articles describing the concealment of the allocation plan in detail, and 13 articles not using the blinding, which may be due to the unclear random allocation method in the study and the subjective bias of insomnia patients and physicians.

At present, the treatment methods for insomnia include drug therapy and cognitive behavioral therapy. Drug therapy can obtain short-term curative effect. If drug use is reduced until stopped, then cognitive behavioral intervention can obtain satisfactory long-term curative effect. The traditional management of drug cognitive therapy is that patients go to the hospital to treat face-to-face with doctors. However, due to the influence of time and geographical factors, there will be problems such as patients withdrawing from treatment and unsatisfactory treatment effect. However, because of the flexibility in time and space, networked cognitive behavioral therapy is more easily accepted by patients. 


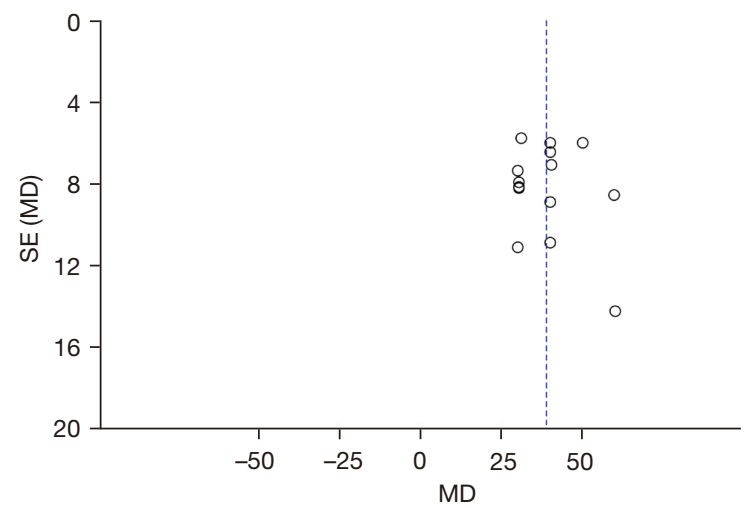

Figure 13 Funnel chart for bias of publication of the included articles for total sleep time. MD, mean difference; SE, Standard Error.

The findings of the present study indicated that the SOL after ICBT was significantly shorter than the SOL in the control group (MD: $-12.27,95 \%$ CI: -16.43 to -9.90 , $\mathrm{P}<0.01)$, the wake-up time after ICBT was significantly shorter in contrast to the control group (MD: -16.91 , 95\% CI: -19.89 to $-13.94, P<0.01$ ), and TIB after ICBT was significantly shorter than the control group (MD: -16.94 , 95\% CI: -24.16 to $-9.71, \mathrm{P}<0.01)$. Improvements in SOL, WASO, and TIB of patients after ICBT may be related to sleep restriction (14-16). The TST after ICBT was significantly longer than that in the control group (MD: 38.67, 95\% CI: $34.70-42.65, \mathrm{P}<0.01)$. The SE after ICBT was significantly higher than that of the control group (MD: 13.28, 95\% CI: $10.49-16.06, \mathrm{P}<0.01)$. The improvement of ICBT on sleep changed the total sleep time of patients. It could also be that insomniacs' subjective perception of increasing their sleep duration affected sleep efficiency $(17,18)$. The NOA after ICBT was significantly lower than that of the control group (OR: $0.33,95 \%$ CI: 0.17 to -0.67 , $\mathrm{P}<0.01$ ), indicating that the improvement in NOA may be related to sleep restriction and stimulation control in ICBT.

It was found that the ISI after ICBT was significantly smaller than that of the control group (MD: $-6.34,95 \%$ CI: -8.24 to $-4.44, \mathrm{P}<0.01)$. The improvement in sleep is related to ISI and CBT, which can aid in sleep $(19,20)$.

In addition, a meta-analysis was performed on the anxiety and depression levels of insomnia patients. The results showed that the anxiety level after ICBT was significantly lower than that of the control group (SMD: $-0.56,95 \%$ CI: -0.84 to $-0.29, \mathrm{P}<0.01)$. The depression level after ICBT was significantly lower than that of the control group
(SMD: $-0.83,95 \%$ CI: -0.94 to $-0.71, \mathrm{P}<0.01$ ). The above data showed that ICBT not only has a good effect on sleep parameters but also reduces the anxiety and depression levels associated with insomnia patients (21). The most common conditions among insomnia patients are anxiety and depression, which can also aggravate insomnia $(22,23)$. ICBT has a significant effect on the anxiety and depression associated with insomnia, which may be related to the CBT and relaxation therapy used in the protocol. By improving the anxiety and depression of the patients, the sleep quality of the patients can also be improved (24).

The network management platform for insomniacs is constructed to implement network management for patients. The platform has the functions of online communication of words, languages, and videos among doctors, nurses, and patients, remote data transmission and offline data application. The platform is provided with a management terminal and a patient terminal. Patients can rent a tablet computer provided by the hospital to download the patient terminal, and remotely manage drug cognitive behavioral therapy through a mobile tablet computer. At present, due to the shortage of medical staff, the time of communication with patients during working hours is limited, and patients have long queues and short communication time with medical staff, which requires a lot of ineffective time. The network management platform can realize online communication, remote data transmission, and offline data access for medical patients. Patients can receive personalized guidance from medical staff anytime and anywhere according to their own time schedule, and learn about sleeprelated knowledge and their own treatment trends. The effectiveness of network cognitive behavioral therapy is strong, and the records are timely and complete to ensure the curative effect.

Meta-analysis was performed to compare the efficacy of ICBT and traditional face-to-face CBT on patients with neurological insomnia. The funnel chart showed that there was no bias in publication, and the conclusions obtained were credible, indicating that the risk of bias was not the main factor affecting the conclusion of this study.

\section{Conclusions}

Fourteen relevant articles on ICBT and face-to-face CBT treatment of patients with neurological insomnia were included in the present study. A meta-analysis was performed to confirm that ICBT can significantly improve 
SOL, TST, SE, WASO, TIB, NOA, and ISI in patients with insomnia, and ICBT had a relieving effect on patients with anxiety and depression. The limitation of the present study was that some of the included articles had certain degrees of heterogeneity, which may have affected the results. Therefore, further research is needed in future work. The present study provided a reliable scientific reference for the treatment of insomnia at our institution.

\section{Acknowledgments}

Funding: This work was funded by grants from Sanming Project of Medicine in Shenzhen (No. SZSM201911003).

\section{Footnote}

Reporting Checklist: The authors have completed the PRISMA reporting checklist. Available at https://dx.doi. org/10.21037/apm-21-2387

Conflicts of Interest: All authors have completed the ICMJE uniform disclosure form (available at https://dx.doi. org/10.21037/apm-21-2387). The authors have no conflicts of interest to declare.

Ethical Statement: The authors are accountable for all aspects of the work in ensuring that questions related to the accuracy or integrity of any part of the work are appropriately investigated and resolved.

Open Access Statement: This is an Open Access article distributed in accordance with the Creative Commons Attribution-NonCommercial-NoDerivs 4.0 International License (CC BY-NC-ND 4.0), which permits the noncommercial replication and distribution of the article with the strict proviso that no changes or edits are made and the original work is properly cited (including links to both the formal publication through the relevant DOI and the license). See: https://creativecommons.org/licenses/by-nc-nd/4.0/.

\section{References}

1. Hedman E, Ljótsson B, Lindefors N. Cognitive behavior therapy via the Internet: a systematic review of applications, clinical efficacy and cost-effectiveness. Expert Rev Pharmacoecon Outcomes Res 2012;12:745-64.

2. Wagner $\mathrm{B}$, Horn $\mathrm{AB}$, Maercker A. Internet-based versus face-to-face cognitive-behavioral intervention for depression: a randomized controlled non-inferiority trial. J Affect Disord 2014;152-154:113-21.

3. Andersson G. Internet-based cognitive-behavioral self help for depression. Expert Rev Neurother 2006;6:1637-42.

4. Zhou T, Li X, Pei Y, et al. Internet-based cognitive behavioural therapy for subthreshold depression: a systematic review and meta-analysis. BMC Psychiatry 2016;16:356.

5. Shi X, Buysse DJ, Ritterband LM, et al. Solving insomnia electronically: Sleep treatment for asthma (SIESTA): A study protocol for a randomized controlled trial. Contemp Clin Trials 2019;79:73-9.

6. Buntrock C, Lehr D, Smit F, et al. Guided Internet-Based Cognitive Behavioral Therapy for Insomnia: HealthEconomic Evaluation From the Societal and Public Health Care Perspective Alongside a Randomized Controlled Trial. J Med Internet Res 2021;23:e25609.

7. Ebert DD, Berking $M$, Thiart $\mathrm{H}$, et al. Restoring depleted resources: Efficacy and mechanisms of change of an internet-based unguided recovery training for better sleep and psychological detachment from work. Health Psychol 2015;34S:1240-51.

8. Ritterband LM, Thorndike FP, Gonder-Frederick LA, et al. Efficacy of an Internet-based behavioral intervention for adults with insomnia. Arch Gen Psychiatry 2009;66:692-8.

9. Webb CA, Rosso IM, Rauch SL. Internet-Based Cognitive-Behavioral Therapy for Depression: Current Progress and Future Directions. Harv Rev Psychiatry 2017;25:114-22.

10. Büscher R, Torok $M$, Terhorst $Y$, et al. Internet-Based Cognitive Behavioral Therapy to Reduce Suicidal Ideation: A Systematic Review and Meta-analysis. JAMA Netw Open 2020;3:e203933.

11. Kyrios M, Ahern C, Fassnacht DB, et al. TherapistAssisted Internet-Based Cognitive Behavioral Therapy Versus Progressive Relaxation in Obsessive-Compulsive Disorder: Randomized Controlled Trial. J Med Internet Res 2018;20:e242.

12. Otte C. Cognitive behavioral therapy in anxiety disorders: current state of the evidence. Dialogues Clin Neurosci 2011;13:413-21.

13. Kaldo-Sandström V, Larsen HC, Andersson G. Internetbased cognitive-behavioral self-help treatment of tinnitus: clinical effectiveness and predictors of outcome. Am J Audiol 2004;13:185-92.

14. Suzuki E, Tsuchiya M, Hirokawa K, et al. Evaluation of an internet-based self-help program for better quality of sleep among Japanese workers: a randomized controlled trial. J 
Occup Health 2008;50:387-99.

15. Retzer L, Feil M, Reindl R, et al. Anonymous online cognitive behavioral therapy for sleep disorders in shift workers-a study protocol for a randomized controlled trial. Trials 2021 Aug 16; 22(1):539.

16. Johansson R, Sjöberg E, Sjögren M, et al. Tailored vs. standardized internet-based cognitive behavior therapy for depression and comorbid symptoms: a randomized controlled trial. PLoS One 2012;7:e36905.

17. Luyster FS, Ritterband LM, Sereika SM, et al. InternetBased Cognitive-Behavioral Therapy for Insomnia in Adults With Asthma: A Pilot Study. Behav Sleep Med 2020;18:10-22.

18. van der Zweerde T, Lancee J, Ida Luik A, et al. InternetDelivered Cognitive Behavioral Therapy for Insomnia: Tailoring Cognitive Behavioral Therapy for Insomnia for Patients with Chronic Insomnia. Sleep Med Clin 2019; 14:301-15.

19. Thiart H, Ebert DD, Lehr D, et al. Internet-Based Cognitive Behavioral Therapy for Insomnia: A Health Economic Evaluation. Sleep 2016;39:1769-78.

20. Chow PI, Ingersoll KS, Thorndike FP, et al. Cognitive mechanisms of sleep outcomes in a randomized clinical

Cite this article as: Yu H, Zhang Y, Liu Q, Yan R. Efficacy of online and face-to-face cognitive behavioral therapy in the treatment of neurological insomnia: a systematic review and meta-analysis. Ann Palliat Med 2021;10(10):10684-10696. doi: 10.21037/apm-21-2387 trial of internet-based cognitive behavioral therapy for insomnia. Sleep Med 2018;47:77-85.

21. Norlund F, Wallin E, Olsson EMG, et al. InternetBased Cognitive Behavioral Therapy for Symptoms of Depression and Anxiety Among Patients With a Recent Myocardial Infarction: The U-CARE Heart Randomized Controlled Trial. J Med Internet Res 2018;20:e88.

22. Newby JM, Smith J, Uppal S, et al. Internet-based cognitive behavioral therapy versus psychoeducation control for illness anxiety disorder and somatic symptom disorder: A randomized controlled trial. J Consult Clin Psychol 2018;86:89-98.

23. Carlbring P, Andersson G, Cuijpers P, et al. Internet-based vs. face-to-face cognitive behavior therapy for psychiatric and somatic disorders: an updated systematic review and meta-analysis. Cogn Behav Ther 2018;47:1-18.

24. Herbst N, Voderholzer U, Thiel N, et al. No talking, just writing! Efficacy of an Internet-based cognitive behavioral therapy with exposure and response prevention in obsessive compulsive disorder. Psychother Psychosom 2014;83:165-75.

(English Language Editor: R. Scott) 